\title{
Le système lymphatique cardiaque
}

> Le système lymphatique est un réseau vasculaire responsable du transport des graisses intestinales. Il participe à la surveillance immune et permet le maintien de l'homéostasie tissulaire. Malgré un grand nombre d'évidences montrant l'importance des vaisseaux lymphatiques dans les maladies cardiovasculaires, le rôle des vaisseaux lymphatiques cardiaques n'a été que très peu étudié. En condition physiologique, le système lymphatique cardiaque régule dynamiquement le drainage des fluides interstitiels vers les ganglions médiastinaux afin de maintenir l'homéostasie du tissu cardiaque et de prévenir la formation d'un œdème. Après un infarctus du myocarde, les vaisseaux lymphatiques du cœur ischémique perdent leur fonction et contribuent au développement d'un œdème myocardique chronique qui aggrave la fibrose et la dysfonction cardiaque. La stimulation de la lymphangiogenèse cardiaque, fondée sur la délivrance de facteurs de croissance lymphangiogéniques comme le VEGF-C, pourrait représenter une nouvelle stratégie thérapeutique pour améliorer la fonction cardiaque. Cette revue met en évidence la chronologie des principales découvertes associées au développement et à la fonction lymphatique cardiaque. <

Le système lymphatique est composé d'un réseau de capillaires et de vaisseaux collecteurs. La fonction principale du réseau lymphatique est le retour, à partir des fluides tissulaires, des lipides, dont le cholestérol, des macromolécules et des cellules immunitaires vers le sang circulant au travers des jonctions lymphaticoveineuses au niveau jugulaire [1]. On évalue entre 5 à 20 litres le volume de lymphe drainée en une journée, en fonction du niveau d'activité et de l'état de santé de l'individu [2]. La lymphe est filtrée sur son passage dans les ganglions lymphatiques où les solutés sont
Barbara Garmy-Susini ${ }^{1}$, Nathalie Pizzinat ${ }^{1}$, Nicole Villeneuve ${ }^{3}$, Antoine Bril ${ }^{3}$, Ebba Brakenhielm², Angelo Parini ${ }^{1}$

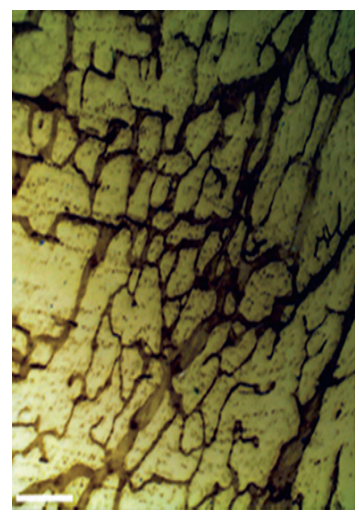

${ }^{1}$ Institut des maladies métaboliques et cardiovasculaires (I2MC), université de Toulouse, Inserm U1048, université Paul Sabatier, 1 , avenue Jean Poulhès, 31432 Toulouse, France ; ${ }^{2}$ Université de Rouen, Inserm U1096, Rouen, France ;

${ }^{3}$ Institut de recherches Servier, Suresnes, France.

angelo.parini@inserm.fr

captés par des cellules présentatrices d'antigènes (CPA) afin d'initier des réponses immunitaires spécifiques contre des particules qui pourraient être pathogènes (Figure 1) [3]. Les capillaires lymphatiques sont présents dans la peau et dans la plupart des organes internes, à l'exception de la moelle osseuse et des tissus avasculaires comme le cartilage, la cornée et l'épiderme.

Dans l'intestin, les vaisseaux lymphatiques ont été initialement nommés «veines blanches » en raison de leur coloration blanche observée en phase postprandiale dans la région mésentérique. Ils jouent un rôle primordial dans l'absorption des lipides intestinaux; ils sont encore appelés «lactés» ou «lactéaux», en raison de cette particularité [4]. Majoritairement présents dans les villosités de l'intestin grêle, les vaisseaux lymphatiques sont à l'origine de l'absorption des lipides alimentaires, libérés sous la forme de chylomicrons par les entérocytes (Figure 1). Les vaisseaux lymphatiques jouent également un rôle important dans le transport réverse du cholestérol $[5,6]$. La composition protéique de la lymphe est équivalente à celle du fluide interstitiel qui est similaire, mais généralement moins concentrée, à celle du plasma sanguin, à l'exception de la lymphe intestinale qui contient une grande quantité de lipides intestinaux [7]. Cette lymphe est une émulsion trouble et laiteuse, souvent appelée «chyle». Le drainage lymphatique peut être considérablement modifié à la suite d'une infection, d'un traumatisme, d'une chirurgie, d'une transplantation, d'un traitement, ou d'une maladie veineuse ou congénitale [8, 9].

La lymphangiogenèse, processus dirigeant la croissance de nouveaux vaisseaux lymphatiques, apparaît au cours du développement embryonnaire. Elle se met en place à partir de bourgeonnements du système sanguin [1]. Elle est également impliquée dans de nombreux états pathologiques (Figure 1). 


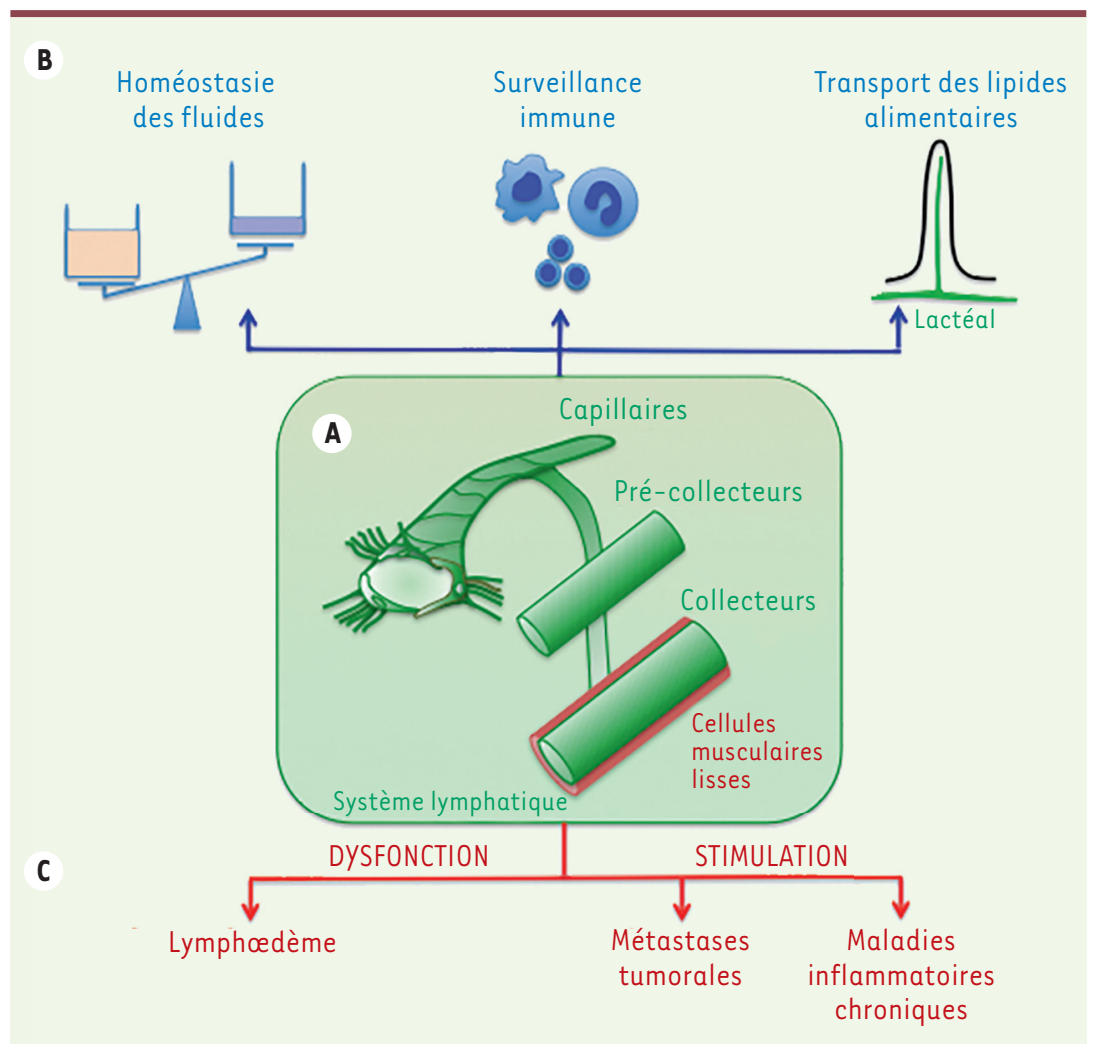

Figure 1. Le système lymphatique. A. Le système lymphatique est composé d'un réseau de capillaires qui fusionnent en un réseau de vaisseaux pré-collecteurs, puis collecteurs, qui se distinguent par la présence de valves et une couverture de cellules musculaires lisses. $\boldsymbol{B}$. Le système lymphatique joue un rôle primordial en physiologie ; il régule la balance des fluides et maintient l'homéostasie tissulaire ; il participe à la surveillance immune et assure le drainage des lipides intestinaux dans les villosités de l'intestin grêle. C. En condition pathologique, un défaut du drainage lymphatique conduit à la formation d'un lymphœdème. En revanche, un excès de lymphangiogenèse est observé lors de la dissémination métastatique et au cours de maladies inflammatoires chroniques.

\section{Origine embryonnaire du système lymphatique}

En 1902, Florence Sabin ${ }^{1}$ propose ce qui sera le modèle le plus largement accepté de développement de la vascularisation lymphatique. À l'aide d'un colorant injecté dans des embryons de porcs, elle met en évidence une origine veineuse des cellules endothéliales lymphatiques (CEL) qui vont former les sacs lymphatiques primaires. À partir de ces structures, les cellules migrent vers les tissus périphériques et s'organisent en vaisseaux. Plus précisément, deux sacs lymphatiques (ou bourgeons) se développent, dans un premier temps, à la jonction des veines sous-clavières et cardinales antérieures. Les vaisseaux lymphatiques formés dans ces sacs se développent ensuite dans la tête, le cou, le thorax et les membres antérieurs de l'embryon. Dans un second temps, des sacs lymphatiques se développent à partir de la veine mésonéphrique et des veines situées à la limite

${ }^{1}$ Florence Sabin (1871-1953) fut la première professeure à diriger un département au Rockefeller institute for medical research, New York, États-Unis. dorsomédiale du canal de Wolff². Ils vont permettre la vascularisation lymphatique de l'abdomen et du diaphragme. Enfin les sacs lymphatiques postérieurs, issus de la jonction de la veine iliaque primitive et de la veine cardinale postérieure, fournissent en vaisseaux lymphatiques la région pelvienne et les membres postérieurs [10]. Dans le cœur, le développement lymphatique apparaît vers le $3^{\mathrm{e}}$ mois du développement embryonnaire chez I'homme, peu de temps après l'apparition d'une circulation coronaire. Le réseau lymphatique se développe d'abord sur les surfaces externe et interne du cœur, de la base vers l'apex, suivi par l'irrigation lymphatique du myocarde. Des études réalisées chez la souris ont montré que l'inhibition de la lymphangiogenèse au cours du développement conduit à un œdème cardiaque dans la période périnatale, démontrant le rôle clé des lymphatiques dans la physiologie cardiaque.

Les récents travaux de Paul Riley [11] remettent en question l'origine veineuse stricte des cellules endothéliales lymphatiques cardiaques. Ils révèlent une origine embryonnaire hétérogène des populations cellulaires. Elles dérivent, d'une part, de l'endothélium veineux extra-cardiaque et ont, d'autre part, une origine progénitrice endothéliale provenant du sac vitellin [12]. La lymphangiogenèse postnatale $a$, par définition, été supposée provenir de vaisseaux lymphatiques préexistants. Cependant, des études récentes montrent que ce processus chez l'adulte pourrait avoir une origine non endothéliale [13]. En raison du manque d'approches de traçage de lignage, ce domaine de recherche reste encore en cours d'investigation. Les lignées myéloïdes ne paraissent cependant pas contribuer à la lymphangiogenèse dans la peau ou le mésentère pendant l'embryogenèse $[14$, 15]. L'hétérogénéité de l'origine du système lymphatique chez l'adulte, et dans différents tissus, est particulièrement intéressante. Elle suggère en effet des mécanismes spécifiques de régulation de la lymphangiogenèse en situations physiologiques et pathologiques.

\footnotetext{
${ }^{2}$ Le canal de Wolff est le canal rénal du pronéphros des embryons de vertébrés.
} 


\section{Pathologies associées au système lymphatique}

Une dysfonction du système lymphatique provoque l'apparition de lymphœdèmes des membres associés à une accumulation de graisses et de lymphe dans les tissus [16]. Un lymphœdème se traduit par un défaut du retour de la lymphe vers la circulation sanguine que l'on met en évidence, par lymphographie, lors du diagnostic. Les lymphœdèmes s'observent majoritairement au niveau des membres inférieurs ou supérieurs. Ils peuvent également, mais plus rarement, affecter d'autres territoires (le cou, l'abdomen, le scrotum, etc.).

Le lymphœdème peut être primaire, ou primitif, associé à une mutation génétique, ou secondaire, à la suite d'une filariose lymphatique ou d'une exérèse tumorale associée à un curage ganglionnaire. La plupart des pathologies associées au système lymphatique sont caractérisées par une stimulation accrue de la lymphangiogenèse. $\varepsilon n$ condition tumorale, le système lymphatique se développe sur le pourtour des tumeurs mais également dans les ganglions lymphatiques sentinelles, participant ainsi à la formation de la niche métastatique [17]. Les vaisseaux lymphatiques représentent la voie majeure de dissémination des tumeurs solides. On observe également une stimulation de la lymphangiogenèse dans les maladies inflammatoires chroniques [3]. Des études récentes ont ainsi confirmé un drainage lymphatique altéré dans la maladie de Crohn, le faisant apparaître comme un acteur critique dans les maladies inflammatoires chroniques de l'intestin [18]. Le système lymphatique, très présent dans le derme, participe également activement au développement de pathologies inflammatoires comme le psoriasis [19] (Figure 1).

La lymphangiogenèse est stimulée par des facteurs de croissance lymphangiogéniques, comme les VEGF(vascular endothelial growth factor)-C et -D. Ces facteurs subissent des maturations post-traductionnelles et protéolytiques avant de se lier à leur récepteur, le VEGFR-3 (un récepteur à activité tyrosine kinase), exprimé à la surface des cellules endothéliales lymphatiques (CEL) [20-22]. Le VEGF-C est indispensable au développement embryonnaire des vaisseaux lymphatiques à partir de la formation des sacs lymphatiques [23]. Son invalidation, chez la souris, conduit à la formation d'œdèmes et à une létalité embryonnaire entre $\varepsilon 15,5$ et $\varepsilon 17,5$ [23]. II stimule la prolifération et la différenciation des $C \varepsilon L$ et est majoritairement synthétisé par les macrophages et les cellules tumorales [24]. Le VEGF-C se lie également au VEGFR-2. Malgré une expression ubiquitaire du VEGF-D, ce facteur n'est pas indispensable à la formation des vaisseaux lymphatiques. Son rôle semble restreint au contrôle de la vasodilatation des vaisseaux lymphatiques au cours des pathologies inflammatoires $[20,25]$.

\section{Système lymphatique et muscle cardiaque}

\section{Un peu d'histoire}

Une relation étroite existe entre le système lymphatique et le muscle cardiaque. Bien que le système lymphatique cardiaque ait été décrit initialement au XVII ${ }^{e}$ siècle par le naturaliste suédois

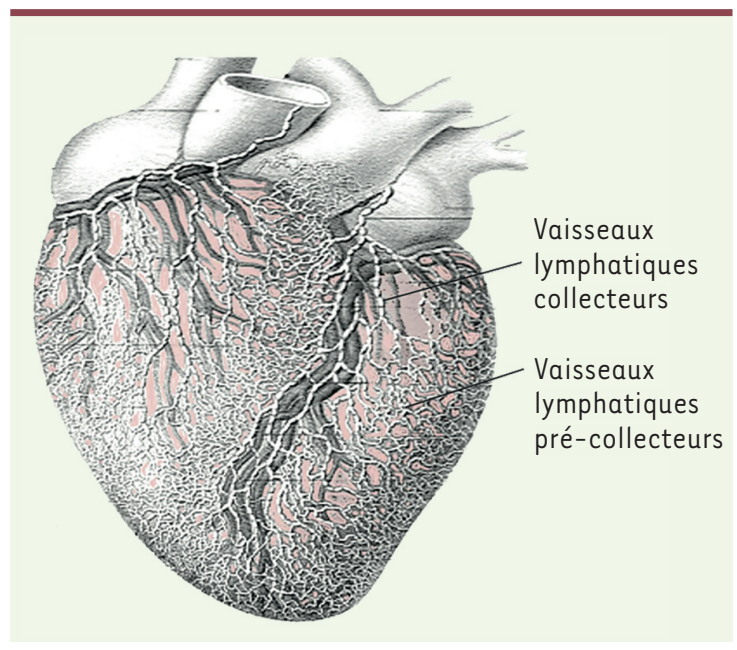

Figure 2. Représentation schématique de l'anatomie du système lymphatique cardiaque humain. Le réseau capillaire intra-myocardique fusionne en un réseau pré-collecteur sousépicardique avant de collecter les fluides interstitiels dans les vaisseaux collecteurs qui longent les artères coronaires, afin de diriger la lymphe vers les ganglions lymphatiques médiastinaux (BIU Santé Paris) (d'après C. Sappey, 1874).

Olaus Rudbeck (1630-1702), il a fallu attendre le $x I x^{e}$ et le $x x^{e}$ siècles pour voir émerger une cartographie de ce système dans le cœur. En 1874, l'anatomiste francais Constant Sappey publie un recueil sur l'anatomie des vaisseaux lymphatiques chez l'homme, montrant la richesse du réseau lymphatique cardiaque (Figure 2). La présence des plexus lymphatiques subendocardiques, myocardiques et subépicardiques, est ensuite démontrée par Sigfred Aagaard, en 1924, par l'injection de colorant dans le muscle cardiaque. Ces découvertes ont été confirmées en 1939 par les recherches de Paul R. Patek (university of California, Los Angeles, UCLA, ÉtatsUnis) qui met en évidence, chez le chien, le drainage lymphatique cardiaque grâce à l'injection dans le myocarde d'encre de Chine. À partir des années 1960, plusieurs études sont réalisées chez de gros animaux, démontrant que le réseau lymphatique cardiaque est essentiel pour l'homéostasie fluidique du cœur et que l'œdème cardiaque, lié à une évacuation lymphatique insuffisante, conduit à la dysfonction cardiaque $[26,27]$.

\section{Description anatomique}

Trois plexus lymphatiques sont responsables du sens de circulation de la lymphe cardiaque qui débute dans le plexus subendocardique et se dirige vers le plexus myocardique, puis le plexus subépicardique [28]. 


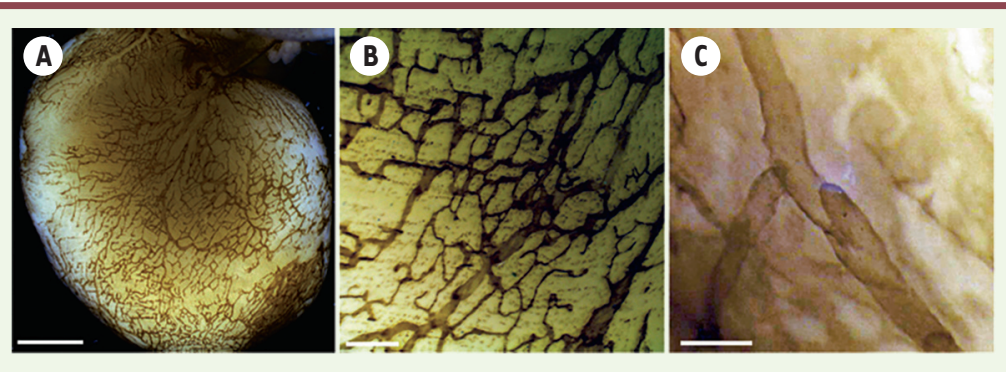

Figure 3. Plexus lymphatique subépicardique. A. Immunodétection du plexus lymphatique subépicardique sur un cœur entier de rat après marquage du VEGF-C (vascular endothelial growth factor-C) spécifique des cellules endothéliales lymphatiques (échelle : $500 \mu \mathrm{m}$ ). B. Le plexus lymphatique subépicardique se présente comme un réseau constitué de vaisseaux pré-collecteurs et de vaisseaux collecteurs (échelle: $50 \mu \mathrm{m}$ ). C. Grossissement montrant une valve de vaisseau collecteur (échelle : $20 \mu \mathrm{m}$ ).

Le drainage s'effectue de la base vers l'apex du cœur au cours de chaque cycle cardiaque. En effet, une particularité des vaisseaux précollecteurs et collecteurs cardiaques est l'absence quasi totale de couches musculaires lisses qui enrobent ces vaisseaux dans les autres tissus. Le drainage lymphatique cardiaque dépend donc de l'activité contractile des cardiomyocytes qui les entourent, afin de propulser la lymphe hors du muscle cardiaque.

Le plexus subendocardique est composé à la fois de larges vaisseaux dilatés et de capillaires. Bien que leur fonction n'ait pas encore été clairement définie, il est cependant établi que ce réseau très interconnecté fait progresser la lymphe vers le plexus myocardique. Ce dernier est essentiellement composé de capillaires répartis de façon éparse en comparaison avec les capillaires sanguins. Ces capillaires ne possèdent pas de membrane basale, ce qui facilite et permet d'ajuster en permanence le passage transendothélial des fluides et des cellules immunitaires comme les lymphocytes $T$ et les cellules présentatrices d'antigènes [29]. Ils se localisent dans l'espace interstitiel, à proximité des capillaires sanguins, et ont une structure en «panier». Ils permettent le drainage des fluides vers des vaisseaux pré-collecteurs avant de rejoindre le plexus subépicardique [30].

Le plexus subépicardique est un plexus intermédiaire formé de vaisseaux lymphatiques pré-collecteurs et collecteurs comportant des valves lymphatiques (Brakenhielm et al., resultats non publiés) (Figure 3). Ces vaisseaux drainent l'ensemble des fluides vers les troncs lymphatiques principaux situés à proximité des artères coronaires gauche et droite [30]. Des vaisseaux lymphatiques sont également retrouvés à la base des valves atrio-ventriculaires. Les hypothèses actuelles proposent l'existence d'une connexion entre ces vaisseaux lymphatiques et ceux situés au niveau des valves aortique et pulmonaire [31]. La présence d'un plexus lymphatique à la base des valves suggère ainsi un rôle potentiel du réseau lymphatique dans la physiopathologie des maladies valvulaires cardiaques, plus précisément au cours de la résolution de l'inflammation et de la fibrose. La densité lymphatique augmente en effet généralement dans les zones riches en matrice extracellulaire. On observe également une lymphangiogenèse accrue dans les valves au cours d'une endocardite infectieuse [32]. La prolifération de vaisseaux lymphatiques à la base des valves pourrait donc participer activement à l'afflux de cellules immunitaires dans l'endocarde, lors de pathologies cardiaques comme l'ischémie aiguë et chronique, l'hypertrophie ou les myocardites.

\section{Système lymphatique et ischémie cardiaque}

Dans le cœur adulte, l'ischémie cardiaque aiguë provoque l'augmentation du transport lymphatique afin de limiter l'œdème résultant de I'hyperperméabilité vasculaire et de cette ischémie. Des travaux réalisés chez l'animal et des données obtenues chez des patients présentant une ischémie cardiaque chronique ou un infarctus du myocarde (IDM), montrent que le réseau lymphatique subit un fort remodelage, avec une perte des vaisseaux précollecteurs (de phénotype lyve $-1^{+}$/ podoplanin ${ }^{+}$) et collecteurs (de phénotype podopla$\mathrm{nin}^{+} / \mathrm{SMA}[\text { smooth muscle actin }]^{+}$), suivie d'une réponse lymphangiogénique (lyve- $1^{+}$) [32]. Ce processus, décrit dans des pathologies inflammatoires cutanées, participe activement à la résolution de l'inflammation.

Des études récentes supportent l'hypothèse d'un drainage lymphatique altéré lors de l'ischémie cardiaque chronique, et la réponse lymphangiogénique postischémique apparaît insuffisante pour prévenir l'œdème myocardique [33]. En effet, un défaut de réabsorption des fluides interstitiels aboutit invariablement à la formation de l'œdème cardiaque. Au-delà d'une augmentation de la pression interstitielle, néfaste pour le cœur, l'œdème contribue également à une modification de la composition des fluides interstitiels avec, notamment, une augmentation des taux protéiques et d'autres macromolécules. Les lymphatiques sont des conduits d'évacuation pour les métabolites générés par le métabolisme cellulaire physiologique et pour les déchets produits lors d'un processus ischémique ou nécrotique. Des études réalisées chez le chien ont montré, après ischémie, une augmentation des taux lymphatiques de potassium, de lactate et de créatinine phosphokinase, ainsi que des métabolites arythmogéniques comme la lysophosphatidylcholine. La participation du système lymphatique dans la réponse immuno-inflammatoire secondaire à l'ischémie cardiaque apparaît évidente. Cependant, cette contribution demeure encore très peu explorée. Un grand nombre de molécules, générées par la mort ou le stress cellulaires, peut être perçu comme des signaux de danger endogènes (ou alarmines). Leur 
drainage par le système lymphatique pourrait réduire l'inflammation aiguë associée à l'ischémie. Le système lymphatique est également un composant important de la réponse immunitaire. Les cellules présentatrices d'antigènes, et les antigènes, transitent vers les ganglions lymphatiques drainants dans lesquels ces cellules participent à l'éducation des cellules de l'immunité adaptive, initiant une réponse immunitaire spécifique ou, au contraire, maintenant une réponse tolérogénique.

La présence de vésicules extracellulaires $(V E)$ a été récemment décrite dans la lymphe [34] et, chez des souris athérosclérotiques, la lymphe est enrichie en $V \varepsilon$. Les vésicules contenues dans la lymphe pourraient donc représenter des biomarqueurs de la dysfonction lymphatique ou de la progression de la maladie inflammatoire.

Chez le rat, un traitement ciblé à base de VEGF-C permet d'accélérer la réponse lymphangiogénique du cœur après un infarctus, avec pour conséquence une réduction de l'œdème cardiaque chronique et une diminution du recrutement des macrophages dans le cœur. Cette amélioration du drainage lymphatique a permis de prévenir la fibrose et a amélioré la fonction cardiaque chez ces animaux [33]. L'ischémie cardiaque provoque la perte de collecteurs lymphatiques dans la région cicatricielle en lien avec le remodelage délétère des vaisseaux pré-collecteurs et l'expansion du réseau lymphatique capillaire sous-épicardique. Ces modifications sont à l'origine d'un transport lymphatique dysfonctionnel résultant en un œdème persistant. Un traitement par le VEGF-C réduit la raréfaction lymphatique et augmente la surface totale de lymphatiques ouverts. Le drainage lymphatique, alors partiellement restauré, participe ainsi à l'élimination des macrophages.

Le système lymphatique cardiaque représente donc un enjeu majeur ouvrant de nouvelles pistes thérapeutiques, en particulier avec le VEGF-C, principal facteur pro-lymphangiogénique, qui pourrait présenter un intérêt dans les maladies cardiovasculaires caractérisées par l'inflammation et l'œdème cardiaques chroniques. $\diamond$

\section{SUMMARY}

\section{Cardiac lymphatic system}

The lymphatic system is a network of vessels and lymphoid tissues that maintain tissue fluid homeostasis, transport intestinal fat, and regulate immune surveillance. Despite a large body of evidence showing the importance of lymphatic vessels in cardiovascular diseases, the role of cardiac lymphatics has not been extensively investigated. This review highlights the chronology of key discoveries in cardiac lymphatic development and function. In physiology, the cardiac lymphatic system dynamically regulates interstitial fluid drainage to the mediastinal lymph nodes to maintain homeostasis and prevent edema. After myocardial infarction, lymphatic vessels in the ischemic heart become dysfunctional and contribute to the development of chronic myocardial edema that aggravates cardiac fibrosis and dysfunction. Stimulation of cardiac lymphangiogenesis, based on the delivery of lymphangiogenic growth factors, such as VEGF-C, may represent a novel therapeutic strategy to improve cardiac function. $\diamond$
LIENS D'INTÉRÊT

Les auteurs déclarent n'avoir aucun lien d'intérêt concernant les données publiées dans cet article.

\section{RÉFÉRENCES}

1. Alitalo K, Tammela T, Petrova TV. Lymphangiogenesis in development and human disease. Nature $2005 ; 438$ : 946-53.

2. Mactier RA, Khanna R, Twardowski Z, et al. Contribution of lymphatic absorption to loss of ultrafiltration and solute clearances in continuous ambulatory peritoneal dialysis. J Clin Invest 1987 ; 80 : 1311-6.

3. Alitalo K. The lymphatic vasculature in disease. Nat Med $2011 ; 17$ : 1371-80.

4. Bernier-Latmani J, Cisarovsky C, Demir CS, et al. DLL4 promotes continuous adult intestinal lacteal regeneration and dietary fat transport. J Clin Invest $2015 ; 125: 4572-86$.

5. Lim Hy, Thiam CH, Yeo KP, et al. Lymphatic vessels are essential for the removal of cholesterol from peripheral tissues by SR-BI-mediated transport of HDL. Cell metab $2013 ; 17: 671-84$.

6. Huang LH, Elvington A, Randolph GJ. The role of the lymphatic system in cholesterol transport. Front pharmacol $2015 ; 6: 182$.

7. Swartz MA. The physiology of the lymphatic system. Adv Drug Deliv Rev $2001 ; 50: 3-20$

8. Swartz MA, Skobe M. Lymphatic function, lymphangiogenesis, and cancer metastasis. Microsc Res Tech 2001 ; 55 : $92-9$.

9. Dietrich T, Bock F, Yuen D, et al. Cutting edge: lymphatic vessels, not blood vessels, primarily mediate immune rejections after transplantation. J immunol $2010 ; 184: 535-9$.

10. Yang $Y$, Oliver $G$. Development of the mammalian lymphatic vasculature. J Clin Invest $2014 ; 124: 888-97$.

11. Klotz L, Norman S, Vieira JM, et al. Cardiac lymphatics are heterogeneous in origin and respond to injury. Nature $2015 ; 522: 62-7$.

12. Norman S, Riley PR. Anatomy and development of the cardiac lymphatic vasculature: Its role in injury and disease. Clin Anat $2016 ; 29$ : 305-15.

13. Aspelund A, Robciuc MR, Karaman $S$, et al. lymphatic system in cardiovascular medicine. Circ Res 2016; 118 : 515-30.

14. Stanczuk L, Martinez-Corral I, Ulvmar MH, et al. cKit lineage hemogenic endothelium-derived cells contribute to mesenteric lymphatic vessels. Cell rep 2015.

15. Martinez-Corral I, Ulvmar MH, Stanczuk L, et al. Nonvenous origin of dermal lymphatic vasculature. Circ Res $2015 ; 116$ : 1649-54.

16. Mortimer PS, Rockson SG. New developments in clinical aspects of lymphatic disease. J Clin Invest 2014 ; 124 : 915-21.

17. Garmy-Susini B, Varner JA. Roles of integrins in tumor angiogenesis and lymphangiogenesis. Lymphat Res Biol 2008 ; 6 : 155-63.

18. von der Weid Py, Rehal S, Ferraz JG. Role of the lymphatic system in the pathogenesis of Crohn's disease. Curr Opin gastroenterol $2011 ; 27: 335-41$.

19. Huggenberger R, Ullmann S, Proulx ST, et al. Stimulation of lymphangiogenesis via VEGFR-3 inhibits chronic skin inflammation. J Exp Med 2010; $207: 2255-69$.

20. Morfoisse F, Tatin F, Hantelys F, et al. Nucleolin promotes heat shockassociated translation of VEGF-D to promote tumor lymphangiogenesis. Cancer Res $2016 ; 76: 4394-405$.

21. Morfoisse F, Kuchnio A, Frainay C, et al. Hypoxia induces VEGF-C expression in metastatic tumor cells via a HIF-lalpha-independent translationmediated mechanism. Cell Rep 2014 ; 6 : 155-67.

22. Bui HM, Enis D, Robciuc MR, et al. Proteolytic activation defines distinct lymphangiogenic mechanisms for VEGFC and VEGFD. J Clin Invest 2016; 126 2167-80.

23. Karkkainen MJ, Haiko P, Sainio K, et al. Vascular endothelial growth factor $C$ is required for sprouting of the first lymphatic vessels from embryonic veins. Nat immunol $2004 ; 5: 74-80$.

24. Dufies M, Giuliano S, Ambrosetti D, et al. Sunitinib stimulates expression of VEGFC by tumor cells and promotes lymphangiogenesis in clear cell renal cell carcinomas. Cancer Res $2017 ; 77$ : 1212-26.

25. Karnezis T, Shayan R, Caesar C, et al. VEGF-D promotes tumor metastasis by regulating prostaglandins produced by the collecting lymphatic endothelium. Cancer Cell 2012 ; 21 : 181-95.

26. Uhley HN, Leeds SE, Sampson JJ, et al. The temporal sequence of lymph flow in the right lymphatic duct in experimental chronic pulmonary edema. Am Heart J $1966 ; 72: 214-7$. 


\section{RÉFÉRENCES}

27. Gloviczki P, Solti F, Szlavy L, Jellinek H. Ultrastructural and electrophysiologic changes of experimental acute cardiac lymphostasis. Lymphology $1983 ; 16: 185-92$.

28. Loukas M, Abel N, Tubbs RS, et al. The cardiac lymphatic system. Clin Anat 2011 ; 24 : 684-91.

29. Bullon A, Huth F. Fine structure of lymphatics in the myocardium. Lymphology $1972 ; 5: 42-8$.

30. Lupinski RW. Aortic fat pad and atrial fibrillation: cardiac lymphatics revisited. ANZ J Surg 2009; $79: 70-4$.

31. Ichikawa S, Uchino S, Hirata Y. Lymphatics of the cardiac chordae tendineae with particular consideration of their origin. Lymphology $1989 ; 22$ : 123-31.

32. Kholova I, Dragneva G, Cermakova P, et al. Lymphatic vasculature is increased in heart valves, ischaemic and inflamed hearts and in cholesterol-rich and calcified atherosclerotic lesions. Eur J Clin Invest $2011 ; 41: 487-97$.
33. Henri 0 , Pouehe $C$, Houssari $M$, et al. Selective stimulation of cardiac lymphangiogenesis reduces myocardial edema and fibrosis leading to improved cardiac function following myocardial infarction. Circulation $2016 ; 133: 1484-97$.

34. Milasan A, Tessandier N, Tan S, et al. Extracellular vesicles are present in mouse lymph and their level differs in atherosclerosis. J Extracell Vesicles $2016 ; 5: 31427$

\section{$15^{\circledR}$ Journées de la Société Française de Myologie}

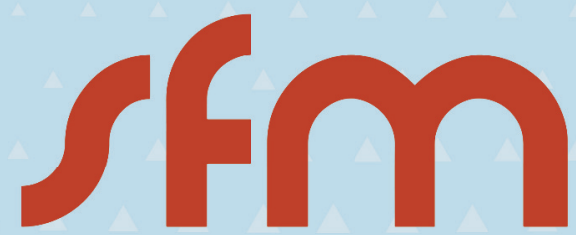

SOCIÉTÉ FRANÇAISE DE MYOLOGIE

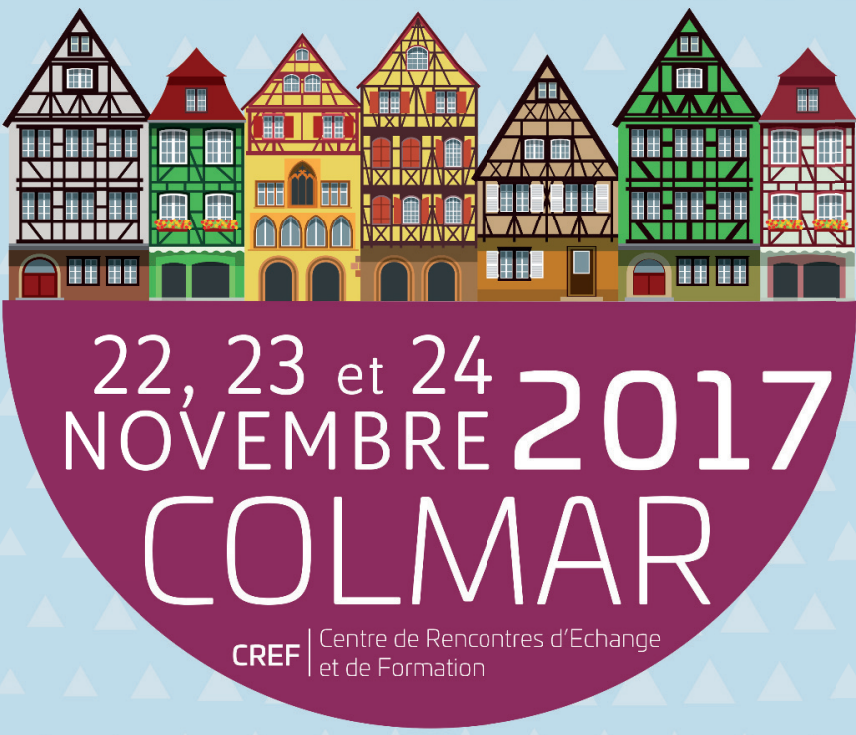

Actualités en myologie

Comité local d'organisation :

Andoni ECHANIZ-LAGUNA (Président) Valérie BIANCALANA

Johann BOHM

Jean-Baptiste CHANSON

Jocelyn LAPORTE

Christine TRANCHANT

TIRÉS À PART

A. Parini 